\title{
On Some Approximation Methods for Solutions of the Dirichlet Problem for Linear Elliptic Equations of Arbitrary Order
}

\author{
FELIX E. BROWDER
}

Communicated by S. BERgMAN

An important tool in the theory of the Dirichlet problem for a single secondorder elliptic partial differential equation is the maximum principle, no analogue of which has yet been found in the more general case of higher order equations or of systems of equations. The portions of the theory of a second-order equation which depend upon the maximum principle in an essential way, such as the Perron-Remak theory of sub-harmonic functions or WIENER's generalized solution of the Dirichlet problem, seem therefore to have no significant generalization to the higher order case. There are certain portions of the classical secondorder theory and of the more special theory of harmonic functions, however, whose development is usually given using the maximum principle but for which the monotonicity properties of the differential operator are not fundamentally relevant and for which a generalization can be given to higher order equations and to systems of equations using the Hilbert space theory of elliptic differential operators. We shall consider several such topics in this paper, the Bergman kernel function, the Schwarz alternating method, and the Poincare "sweeping out" process. Our discussion, which is restricted for the sake of notational simplicity to a single elliptic equation, is valid for the more general class of strongly elliptic systems of differential equations.

In Section 1 we present a summary of the definitions and principal results of the Hilbert space approach to the Dirichlet problem for the general linear elliptic partial differential equation. In Section 2 we establish the existence of kernel functions for such problems as well as their approximability in terms of complete orthonormal sequences of solutions of the homogeneous equation. Section 3 is devoted to the Schwarz alternating method which is considered as a special case of a more general process of iterative solution of the Dirichlet problem generalizing the Poincaré balayage process.

The writer is indebted to $\mathrm{S}$. KAKUTANI for making him acquainted with the latter's unpublished results on the convergence of iterated projections in Hilbert space which form the basis of our generalization of the balayage process. 
Section 1: Let $G$ be an open set in Euclidean $n$-space with points $x=\left(x_{1}, \cdots, x_{n}\right), D_{j}=\partial / \partial x_{i}, C_{c}^{\infty}(G)$ the family of all complex-valued infinitely differentiable functions with compact support in $G$. For a multi-index $\alpha=$ $\left(\alpha_{1}, \cdots, \alpha_{r}\right), 1 \leqq \alpha_{i} \leqq n$, we set $|\alpha|=r$ while $D^{\alpha}=D_{\alpha_{1}} \cdots D_{\alpha_{r}} .(D \emptyset=$ the identity if $\emptyset$ is the empty set, $|\emptyset|=0$.)

$L^{2}(G)$ is the Hilbert space of equivalence classes of complex-valued squareintegrable functions on $G$ with the usual inner product, $(u, v)=\int_{G} u(x) \overline{v(x)} d x$ ( $\bar{v}$ the complex conjugate of $v$, integration with respect to Lebesgue $n$-measure). $C^{i}(G)$ is the family of $j$-times continuously differentiable functions in $G, C^{i}(\bar{G})$ the subfamily of $C^{i}(G)$ of those $f$ such that $D^{\alpha} f$ is continuous on the closure of $G$ for all $\alpha$ with $|\alpha| \leqq j$.

Definition 1: $W^{m, 2}(G)=\left\{u \mid u \varepsilon L^{2}(G)\right.$; the distribution derivative $D^{\alpha} u \varepsilon L^{2}(G)$ for every $\alpha$ with $|\alpha| \leqq m\}$.

For every $j$ with $0 \leqq j \leqq m$ we may define a form $(u, v)_{j}$ for $u$ and $v$ in $W^{m, 2}(G)$, which is linear in $u$ and conjugate linear in $v$, by

$$
(u, v)_{i}=\sum_{|\beta|=-i}\left(D^{\beta} u, D^{\beta} v\right)
$$

If we define an inner product on $W^{m, 2}(G)$ by

$$
[u, v]=\sum_{i=0}^{m}(u, v)_{i}
$$

it is easy to see that $W^{m, 2}(G)$ is a Hilbert space with respect to this inner product with norm $\|u\|=[u, u]^{\frac{1}{2}}$. For each $j$ with $0 \leqq j \leqq m$ we may define the $j$-seminorm on $W^{m, 2}(G)$ by $\|u\|_{i}=(u, u)_{i}^{\frac{1}{2}}$.

Definition 2: $\dot{W}^{m, 2}(G)$ is the closure of $C_{c}^{\infty}(G)$ in $W^{m, 2}(G)$.

Suppose a function $u$ lies in $W^{m, 2}(G) \cap C^{m-1}(G)$ and has null Dirichlet data of order $m$ on the boundary of $G$, i.e. for every $\epsilon>0$ there exists a compact set $C_{\epsilon}$ contained in $G$ such that $\left|D^{\beta} u\right|<\epsilon$ on $G-C_{\epsilon}$ for all $|\beta| \leqq m-1$. Then $u$ lies in $W^{m, 2}(G) \cdot \dot{W}^{m, 2}(G)$ will be described as the family of functions in $W^{m, 2}(G)$ whose Dirichlet data of order $m$ vanishes on the boundary of $G$ in the variational sense. (A general definition of Dirichlet data for functions in $W^{m, 2}(G)$ will be described in Section 3 below, from which this terminology will derive a more literal descriptiveness.)

Let $A$ be a partial differential operator of order $2 m(m \geqq 1)$ with coefficients defined on $G$. For the sake of simplicity we shall assume throughout that $A$ has infinitely differentiable coefficients in $G$, although the results depend only upon a very limited amount of differentiability of the coefficients. $A$ may be written (in general, not uniquely) in the form:

$$
A u=\sum_{|\alpha|,|\beta| \leqq m} D^{\beta}\left(a_{\alpha \beta}(x) D^{\alpha} u\right),
$$


where the $a_{\alpha \beta}$ are infinitely differentiable real-valued functions on $G$. The characteristic form $a(x, \xi)$ of the differential operator $A$ is defined by

$$
a(x, \xi)=\sum_{|\alpha|,|\beta|=m} a_{\alpha \beta}(x) \xi^{\alpha} \xi^{\beta},
$$

where $\xi$ is a real $n$-vector $\left(\xi_{1}, \cdots, \xi_{n}\right), \xi^{\alpha}=\xi_{\alpha_{1}} \cdots \xi_{\alpha_{m}}$, and $x$ is a point of $G$. $A$ is said to be elliptic at $x$ if there exists a constant $c(x)$ such that $a(x, \xi) \geqq$ $c(x) \sum_{|\beta|=m}\left(\xi^{\beta}\right)^{2}$, elliptic in $G$ if it is elliptic for every point of $G$, uniformly elliptic in $G$ if in addition there exists $c_{0}>0$ such that $c(x) \geqq c_{0}$ for all $x$ in $G$.

If $f$ and $g$ are two given smooth functions on $G$, the Dirichlet problem in the strict sense asks for a solution $u$ in $C^{2 m}(G)$ of the equation $A u=f$ in $G$ such that $u-g$ has null Dirichlet data in the strict sense on the boundary of $G$. We may transform this problem into another problem concerning the solution of an operator equation in the Hilbert space $\dot{W}^{m, 2}(G)$ (BRowDER [2], [3]; GÅRDING [8]; VisıK [16]).

Corresponding to the formula (3) for $A$, we define the Dirichlet form $\alpha(u, v)$ for $u, v$ in $W^{m, 2}(G)$ :

$$
a(u, v)=\sum_{|\alpha|,|\beta| \leqq m}(-1)^{|\beta|}\left(a_{\alpha \beta} D^{\alpha} u, D^{\beta} v\right) .
$$

Suppose we are given a function $u \varepsilon C^{2 m}(G) \cap W^{m, 2}(G)$ for which $A u=f$ with $u-g$ having null Dirichlet data on the boundary of $G, f \varepsilon L^{2}(G), g \varepsilon W^{m, 2}(G)$. If we form the equation $(A u, \phi)=(f, \phi)$ for $\phi \varepsilon C_{c}^{\infty}(G)$ and integrate by parts, we obtain

$$
a(u, \phi)=(f, \phi), \quad u-g \varepsilon \dot{W}^{m, 2}(G) .
$$

Definition 3: A function $u$ in $W^{m, 2}(G)$ is said to be a solution of the Dirichlet problem in the variational sense with inhomogeneous part $f$ and boundary function $g$ provided it satisfies condition (5).

Let $A^{+}$be the formal adjoint differential operator to $A$ :

$$
A^{+} u=\sum_{|\alpha|,|\beta| \leqq m}(-1)^{|\alpha|+|\beta|} D^{\alpha}\left(a_{\alpha \beta}(x) D^{\beta} u\right) .
$$

Every solution of (5) satisfies the equation $\left(u, A^{+} \phi\right)=(f, \phi)$ for $\phi$ in $C_{c}^{\infty}(G)$, i.e. is a distribution solution of $A u=f$. Let $r_{n}=\left[\frac{1}{2} n\right]$. It has been shown for $A$ elliptic that if $f \varepsilon W^{r, 2}(G)$ for $r \geqq r_{n}+1$, then $u \varepsilon C^{2 m}(G)$ and $A u=f$ is satisfied in the ordinary sense (BRowder [3], FrIEdrICHs [7]). If we suppose moreover that $A$ is uniformly elliptic on the bounded domain $G$ with the boundary of $G$ composed of surfaces of class $C^{m+r_{n}}, a_{\alpha \beta}$ lie in $C^{r_{n}}(\bar{G}), f \varepsilon W^{r_{n}+1}(G), g \varepsilon$ $W^{r_{n}+m}(G)$, then every solution $u$ of (5) will lie in $C^{m-1}(\bar{G})$ and have the same Dirichlet data in the strict sense as $g$ on the boundary of $G$ (Browder [4], Guseva [9], Nirenberg [12]).

We shall henceforward consider only the Dirichlet problem in the variational sense for an elliptic operator $A$, bearing in mind the results of the last paragraph connecting this problem with the Dirichlet problem in the strict sense. We shall show that (5) is equivalent to an operator equation in $\dot{W}^{m, 2}(G)$. 
By the Schwarz inequality there exists $c_{1}>0$ such that $|a(u, v)| \leqq c_{1}\|u\|\|v\|$ for all $u, v$ in $W^{m, 2}(G)$. Furthermore $|(f, v)| \leqq\|f\|_{0} \cdot\|v\|_{0} \leqq\|f\|_{0}\|v\|$ for $v$ in $W^{m, 2}(G)$. For fixed $u$ in $W^{m, 2}(G), a(u, v)$ is a bounded conjugate-linear form in $v$ for $v$ ranging over the Hilbert space $\dot{W}^{m, 2}(G)$. Thus there exists an unique element $T u \varepsilon \dot{W}^{m, 2}(G)$ such that $[T u, v]=a(u, v)$ for $v \varepsilon \dot{W}^{m, 2}(G) . T$ is a bounded linear mapping of $W^{m, 2}(G)$ into $\dot{W}^{m, 2}(G)$ and $\|T\| \leqq c_{1}$. Similarly there exists an element $C f$ of $\dot{W}^{m, 2}(G)$ such that $[C f, v]=(f, v)$ for all $v$ in $\dot{W}^{m, 2}(G)$ and $C$ is a bounded linear transformation of $L^{2}(G)$ into $\dot{W}^{m, 2}(G)$. (Indeed, $C$ is a bounded linear transformation of $W^{-m, 2}(G)$ into $\dot{W}^{m, 2}(G)$. Cf. [5].) Since $\dot{W}^{m, 2}(G)$ is a linear subset of $L^{2}(G)$, we may also consider $C$ as a bounded linear transformation of $\dot{W}^{m .2}(G)$.

Let $v=u-g$. In terms of $T, C$, and $v$, condition (5) becomes

$$
[T v, \phi]+[T g, \phi]=[C f, \phi] \text { for } \phi \varepsilon C_{c}^{\infty}(G) ; v \varepsilon \dot{W}^{m, 2}(G) \text {. }
$$

Since $C_{c}^{\infty}(G)$ is dense in $\dot{W}^{m, 2}(G)$, this is simply equivalent to $T v=C f-T g$, $v \varepsilon \dot{W}^{m, 2}(G)$. The question as to the solutions of (5), the Dirichlet problem in the variational sense, consists therefore of inverting the operator $T$ on $\dot{W}^{m, 2}(G)$. A particularly simple situation occurs when $G$ satisfies the condition:

$G$ is bounded.

If $A$ is uniformly elliptic on $G$ and if $a_{\alpha \beta} \varepsilon C^{0}(\bar{G})$, it follows from a Fourier transform argument (GÅRING [8], BRowder [26]) that there exist $c_{2}>0$, $k_{0} \geqq 0$ such that:

$$
(-1)^{m} \operatorname{Re} a(u, u) \geqq c_{2}[u, u]-k_{0}(u, u), u \varepsilon \dot{W}^{m, 2}(G) .
$$

Let $T_{1}=(-1)^{m} T+k_{0} C, T$ and $C$ being restricted to $\dot{W}^{m, 2}(G)$. Then (7) is equivalent to $\operatorname{Re}\left[T_{1} u, u\right] \geqq c_{2}\|u\|^{2}$ for $u \varepsilon \dot{W}^{m, 2}(G)$. In particular, $\left\|T_{1} u\right\| \geqq$ $\left[T_{1} u, u\right] /\|u\| \geqq c_{2}\|u\|$ and $T_{1}$ has a bounded inverse $T_{1}^{-1}$ defined on its range, which is a closed subspace of $\dot{W}^{m, 2}(G) . R\left(T_{1}\right)$, the range of $T_{1}$, must equal the whole of $\dot{W}^{n, 2}(G)$ since if $v \varepsilon R\left(T_{1}\right), 0=\operatorname{Re}\left[T_{1} v, v\right] \geqq c_{2}\|v\|^{2}$ implies $v=0$. Thus $T_{1}^{-1}$ is a bounded linear transformation of $\dot{W}^{m, 2}(G)$. Since $T=(-1)^{m} T_{1}+$ $(-1)^{m+1} k_{0} C$, the element $v$ from $\dot{W}^{m, 2}(G)$ is a solution of $T v=C f-T g$ if and only if

$$
T_{1} v-k_{0} C v=(-1)^{m} C f+(-1)^{m+1} T g .
$$

If $\left(\mathrm{g}_{0}\right)$ holds, it follows from a well known lemma of ReLLICH ([3], page 32) that $C$ is a compact linear transformation of $\dot{W}^{m, 2}(G)$. Hence so is $C_{1}=-k_{0} T_{1}^{-1} C$. Applying $T_{1}^{-1}$ to both sides of (8), we see that (8) is equivalent to

$$
v+C_{1} v=(-1)^{m} T_{1}^{-1} C f+(-1)^{m+1} T_{1}^{-1} T g .
$$

We shall restrict ourselves in the remainder of this paper to elliptic differential operators $A$ for which the inequality (7) holds with $k_{0}=0$, i.e. satisfying the condition:

$$
\operatorname{Re} a(u, u) \geqq c_{2}\|u\|^{2}, \quad \dot{u} \varepsilon W^{m, 2}(G) .
$$


This class includes in particular all homogeneous $2 m^{\text {th }}$ order elliptic operators with constant coefficients as well as second-order elliptic operators with nonpositive zero ${ }^{\text {th }}$ order term. Since $\left(\mathrm{g}_{0}\right)$ was used only to ensure the compactness of $C$, and since $\left(\mathrm{p}_{0}\right)$ implies that $C$ may be taken as zero, the Dirichlet problem in the variational sense may be solved under the condition $\left(\mathrm{p}_{0}\right)$ even on unbounded domains. In any case, $C=0$ and the solution $u$ of (5) in this case is given by

$$
u=g+(-1)^{m+1} T_{1}^{-1} T g+(-1)^{m} T_{1}^{-1} C f .
$$

If we set $P=I+(-1)^{m+1} T_{1}^{-1} T$, we see

Definition 4: The function $u=P g$ is the solution of the equation $A u=0$ having the same Dirichlet data in the variational sense on the boundary of $G$ as the prescribed function $g$ in $W^{m, 2}(G)$.

Definition 5: $H$ is the subspace of $W^{m, 2}(G)$ consisting of all solutions $u$ of the equation $A u=0$.

$P$ is a (in general, non-orthogonal) projection of $W^{m, 2}(G)$ onto $H$.

The structure of the subspace $H$ is very special because of the ellipticity of $A$. In particular, all elements of $H$ lie in $C^{\infty}(G)$ and we have the following:

$\left(\mathrm{h}_{1}\right)$ For each multi-index $\alpha$ there is a continuous function $c_{\alpha}(x)$ defined on $G$ such that, for all $u$ in $H$,

([2c], [7]).

$$
\left|D^{\alpha} u(x)\right| \leqq c_{\alpha}(x)\|u\|_{0}
$$

Section 2: Let $\langle u, v\rangle$ be an inner product defined on a subset $F$ of $W^{m, 2}(G)$ with $\|u\|_{F}=\langle u, u\rangle^{\frac{1}{2}}$. We suppose that $F$ contains $C_{c}^{\infty}(G)$ and satisfies the following conditions:

$\left(\mathrm{f}_{1}\right) F$ is a Hilbert space with respect to $\|\cdot\|_{F}$.

$\left(\mathrm{f}_{2}\right)$ There exists $c_{4}>0$ such that, for all $u$ in $F,\|u\| \leqq c_{4}\|u\|_{F}$.

$\left(\mathrm{f}_{3}\right)$ If $\langle u, \phi\rangle=0$ for all $\phi \varepsilon C_{c}^{\infty}(G)$ for a given $u$ in $F$, then $u \varepsilon C^{\infty}(G)$.

Let us remark that any inner product on $W^{m, 2}(G)$ yielding a norm equivalent to $\|\cdot\|$ obviously satisfies conditions $\left(\mathrm{f}_{1}\right)$ and $\left(\mathrm{f}_{2}\right)$. If $\langle u, v\rangle=a(u, v)$ where $a(u, v)$ is a Dirichlet form corresponding to an elliptic differential operator with infinitely differentiable coefficients, then $\left(f_{3}\right)$ is satisfied.

By the property $\left(\mathrm{h}_{1}\right)$ of the subspace $H$ we have for each $x$ in $G$

$$
|P u(x)| \leqq c(x)\|P u\|_{0} \leqq c(x)\|P u\| \leqq c_{3} c(x)\|u\| \leqq c_{3} c_{4} c(x)\|u\|_{F} .
$$

Hence $(P u)(x)$ is a bounded linear functional of $u$ in $F$ and uniquely representable in the form:

$$
(P u)(x)=\left\langle u, k_{x}\right\rangle, \quad u \varepsilon F,
$$

where $k_{x}$ is an element of $F$ depending upon the point $x$ in $G$. By the same argument, for each multi-index $\alpha$ there exists an unique element $k_{x}^{\alpha}$ in $F$ such that

$$
D^{\alpha}(P u)(x)=\left\langle u, k_{x}^{\alpha}\right\rangle, \quad u \varepsilon F
$$


Consider the measurable functions $k(x, y), k^{\alpha}(x, y)$ defined up to a set of measure zero in $G \times G$ by the prescription $k(x, y)=k_{x}(y), k^{\alpha}(x, y)=k_{x}^{\alpha}(y)$. Let $D_{x}^{\alpha}, D_{y}^{\beta}$ denote the partial derivatives in the distribution sense with respect to the $x$ and $y$ variables respectively.

Lemma 1: $D_{x}^{\alpha} k(x, y)=k^{\alpha}(x, y)$.

Proof of Lemma 1: It suffices, first, to show that $D_{x}^{\alpha} k_{x}$ is an element of $F$ and that, for all $u$ in $F, D_{x}^{\alpha}(P u)(x)=\left\langle u, D_{x}^{\alpha} k_{x}\right\rangle$, since $k_{x}^{\alpha}$ is the unique element of $F$ satisfying this last relation. It suffices to show, moreover, that if $g$ is a function from $G$ to $F$ such that $\langle u, g\rangle=s(x)$ where $s(x)$ is a differentiable function in $G$, then $g$ is a weakly differentiable function from $G$ to $F$ and the distribution derivative $D_{i} g$ coincides with the weak derivative in $F$. The weak differentiability of the function $g$ is equivalent to the differentiability of the function $s(x)$. Since the difference quotients converge weakly in $F$ and, since by $\left(\mathrm{f}_{2}\right)$ the injection mapping of $F$ into $W^{m, 2}(G)$ is bounded and hence weakly continuous, they converge to the same limit weakly in $W^{m, 2}(G)$ and hence as distributions. Since the distribution derivative is the limit in the distribution topology of the difference quotients, the two derivatives must be the same.

We know that for $\phi \varepsilon C_{c}^{\infty}(G), P \phi=0$. Therefore $\left\langle\phi, k_{x}^{\alpha}\right\rangle=0$ for all $\alpha$ and, by $\left(\mathrm{f}_{3}\right), D_{y}^{\beta} k^{\alpha}(x, y)$ is continuous in $G$ for every $x$ in $G$ and every $\alpha$ and $\beta$. It follows, from the fact that $D_{x}^{\alpha} D_{y}^{\beta} k(x, y)$ is a locally summable function for every $\alpha$ and $\beta$, that $k(x, y)$ lies (after change on a set of measure zero) in $C^{\infty}(G \times G)$ (Schwartz [13], p. 47). Thus we have

Theorem 1: For each $F$ contained in $W^{m, 2}(G)$ and satisfying conditions $\left(\mathrm{f}_{1}\right)$, $\left(\mathrm{f}_{2}\right)$, and $\left(\mathrm{f}_{3}\right)$ above, there exists a kernel function $k(x, y) \varepsilon C^{\infty}(G \times G)$ such that if $k_{x}(y)=k(x, y), k_{x} \varepsilon F$ for every $x$ in $G$ and $\left\langle u, k_{x}\right\rangle=P u(x)$.

We now specialize the space $F$ and the operator $A$ under consideration. We shall assume that $A$ is formally self-adjoint and has a Dirichlet form satisfying the following conditions:

$$
\begin{gathered}
a(u, v)=a(u, v) ; \quad a(u, u) \geqq c_{5}\|u\|^{2} \text { for } u, v \varepsilon W^{m, 2}(G) \\
\left(c_{5}>0, \text { independent of } u\right) .
\end{gathered}
$$

The prototype of such operators $A$ is the case of the second-order operator $\sum_{i, j} D_{j}\left(a_{i j}(x) D_{i} u\right)+c(x) u, c(x)<-c_{5}$. The properties of the kernel function for operators satisfying condition $\left(\mathrm{p}_{2}\right)$ defined in Section 3 will be discussed in that section and includes the case of $c(x) \equiv 0$.

If $A$ satisfies $\left(\mathrm{p}_{1}\right)$, we choose $F$ to be $W^{m, 2}(G)$ and take $\langle u, v\rangle=a(u, v)$. It is easy to see that conditions $\left(\mathrm{f}_{1}\right),\left(\mathrm{f}_{2}\right)$, and $\left(\mathrm{f}_{3}\right)$ are satisfied. Since $W^{m, 2}(G)$ is separable, $F$ and its closed subspace $H$ are separable Hilbert spaces.

Theorem 2: Suppose that $A$ satisfies $\left(\mathrm{p}_{1}\right), F$ is the whole of $W^{m, 2}(G)$ with the inner product $\langle u, v\rangle=a(u, v)$, and $k(x, y)$ is the kernel function described in Theo- 
rem 1. Let $\left\{\psi_{i}(x)\right\}$ be a complete orthonormal sequence in $H$ with respect to the inner product $\langle u, v\rangle$ and $s_{r}(x, y)=\sum_{i=1}^{r} \overline{\psi_{i}(x)} \psi_{i}(y)$. Then $s_{r}(x, y)$ converges uniformly to $k(x, y)$ on compact subsets of $G \times G$. Similarly for every $\alpha$ and $\beta, D_{x}^{\alpha} D_{y}^{\beta} k(x, y)$ is the uniform limit of $D_{x}^{\alpha} D_{\nu}^{\beta} s_{r}(x, y)$ on every compact subset of $G \times G$.

Proof of Theorem 2: Since $P \phi=0$ for all $\phi \varepsilon C_{c}^{\infty}(G),(12)$ implies that $a\left(k_{x}, \phi\right)=0$ for all such $\phi$. Therefore $k_{x} \varepsilon H$ for each $x$ in $G$. Similarly $D_{x}^{\alpha} k_{x} \varepsilon H$ for every $x$ in $G$ and every multi-index $\alpha$. On the other hand, since $\psi_{i} \varepsilon H, P \psi_{i}=\psi_{i}$. Hence $P \psi_{j}(x)=\psi_{i}(x)=\left\langle\psi_{j}, k_{x}\right\rangle$. Since $k_{x} \varepsilon H$ for every $x$ in $G$, we have $k_{x}=$ $\sum_{i=1}^{\infty} \overline{\psi_{i}(x)} \psi_{i}$, the infinite series converging in $H$. Let $s_{r, x}$ be the element of $H$ defined by $s_{r, x}=\sum_{j=1}^{r} \overline{\psi_{j}(x)} \psi_{i}$. By property $\left(\mathrm{h}_{1}\right)$ of $H$, since the sequence $s_{r, x}$ for fixed $x$ converges strongly to $k_{x}$ in $H$ as $r \rightarrow \infty$, it converges uniformly on every compact subdomain together with all the derivatives $D_{y}^{\beta} s_{r, x}$. Since $s_{r}(x, y)=\overline{s_{r}(y, x)}$ and, for each $x, s_{r}(x, y)$ converges to $k(x, y)$ uniformly in $y$ on compact subsets of $G, k(x, y)=\overline{k(y, x)}$. It follows immediately that, for fixed $y$ in $G, s_{r}(x, y)$ converges to $k(x, y)$ uniformly in $x$ on every compact subset of $G$. Similarly $D_{x}^{\alpha} s_{r}(x, y)$ converges to $D_{x}^{\alpha} k(x, y)$ as $r \rightarrow \infty$ uniformly in $x$ on every compact subset of $G$. Since $D_{x}^{\alpha}\left(P \psi_{i}\right)(x)=D_{x}^{\alpha} \psi_{i}(x)=\left\langle\psi_{i}, D_{x}^{\alpha} k_{x}\right\rangle$, we have $D_{x}^{\alpha} D_{y}^{\beta} s_{r}(x, y)$ converging to $D_{x}^{\alpha} D_{y}^{\beta} k(x, y)$ uniformly for a fixed $x$ in $G$ and $y$ in a compact subset of $G$ or for a fixed $y$ in $G$ and $x$ in a compact subset of $G$.

To complete the proof of the sharper result stated in Theorem 3 we consider a compact subset $K$ of $G$. We have already observed that $D_{x}^{\alpha} k_{x} \varepsilon H$ for every multi-index $\alpha$ and that $D_{x}^{\alpha} \psi_{j}(x)=\left\langle\psi_{j}, D_{x}^{\alpha} k_{x}\right\rangle$. By the Parseval equality $\left\|D_{x}^{\alpha} k_{x}\right\|_{\text {r }}^{2}$ $=\sum_{i=1}^{\infty}\left|D_{x}^{\alpha} \psi_{j}(x)\right|^{2}$. Since $\left|\left\langle u, D_{x}^{\alpha} k_{x}\right\rangle\right|=\left|D_{x}^{\alpha}(P u)(x)\right| \leqq c_{\alpha}^{\prime}(x)\|u\|_{F}$ for all $u$ in $W^{m, 2}(G)$, we have $\left\|D_{x}^{\alpha} k_{x}\right\|_{F} \leqq c_{\alpha}^{\prime}(x)$, where $c_{\alpha}^{\prime}(x)$, which is a constant multiple of the $c_{\alpha}(x)$ of $\left(h_{1}\right)$, is continuous on $G$. Thus $\sum_{j=1}^{\infty}\left|D_{x}^{\alpha} \psi_{i}(x)\right|^{2} \leqq c_{\alpha, 6}^{2}(K)$ for all $x$ in $K$. In particular,

$$
\begin{aligned}
&\left|D_{x}^{\alpha} D_{y}^{\beta} s_{r}(x, y)\right| \leqq\left\{\sum_{j=1}^{r}\left|D_{x}^{\alpha} \psi_{j}(x)\right|^{2}\right\}^{\frac{1}{3}}\left\{\sum_{i=1}^{r}\left|D_{y}^{\beta} \psi_{i}(y)\right|^{2}\right\}^{\frac{1}{3}} \\
& \leqq c_{\alpha, 6}(K) \cdot c_{\beta, 6}(K) ; \quad(x, y) \varepsilon K \times K .
\end{aligned}
$$

It follows that

$$
\iint_{K \times K}\left|D_{x}^{\alpha} D_{y}^{\beta} k(x, y)-D_{x}^{\alpha} D_{y}^{\beta} s_{r}(x, y)\right|^{2} d x d y \rightarrow 0 \quad \text { as } \quad r \rightarrow \infty
$$

since the integrand is uniformly bounded on a set of finite measure and converges to zero everywhere. But then, by a theorem of Sobolev and Schwartz (Schwartz [13], p. 47), $s_{r}(x, y)$ converges uniformly to $k(x, y)$ on $K_{1} \times K_{1}$ where $K_{1}$ is a compact subset of the interior of $K$. The same is true for the derivatives $D_{x}^{\alpha} D_{y}^{\beta} s_{r}(x, y)$, and the theorem is proved.

Section 3: In the present section we shall assume our differential operator $A$ to be formally self-adjoint and such that it can be written in the following 
"homogeneous" form

$$
A u=\sum_{|\alpha|,|\beta|=m} D^{\beta}\left(a_{\alpha \beta}(x) D^{\alpha} u\right) \quad\left(a_{\alpha \beta}=a_{\beta \alpha}\right)
$$

with the corresponding Dirichlet form $a(u, v)$ satisfying the following positivity condition:

$\left(\mathrm{p}_{2}\right) \quad$ There exists a constant $c_{7}>0$ such that $a(u, u) \geqq c\|u\|_{m}^{2}$

$$
\text { for all } u \text { in } W^{m, 2}(G) \text {. }
$$

Let $F_{0}$ be the finite-dimensional subspace of $W^{m, 2}(G)$ spanned by the monomials with total degree less than $m$. If $\left(\mathrm{p}_{2}\right)$ is valid and if for an element $u$ of $W^{m, 2}(G)$ we have $a(u, u)=0$, then $\|u\|_{m}=0$ and $u \varepsilon F_{0}$. Conversely, if $u \varepsilon F_{0}, a(u, v)=0$ for all $v$ in $W^{m, 2}(G)$.

We impose several restrictions upon the domain $G$. The first is

If $S$ is a bounded set in $W^{m, 2}(G), S$ is compact in $W^{m-1,2}(G)$.

It follows from theorems of Sobouev and Kondrashov that $\left(\mathrm{g}_{1}\right)$ is valid for any bounded domain $G$ for which there exist $h>0$ and $a_{0}>0$ such that at every point $x$ of $G$ there exists a right circular cone with vertex at $x$ lying completely in $G$ with height $h$ and cross-sectional area $a_{0}$ (SoboLev [10], KonDRASHOV [15]). In particular, $\left(\mathrm{g}_{1}\right)$ will be satisfied by any domain for which we have

$\left(\mathrm{g}_{2}\right) \quad G$ is a bounded domain with boundary $B(G)$ composed of a finite

$$
\text { number of surfaces of class } C^{m} \text { in } E^{n} \text {. }
$$

It follows from $\left(\mathrm{g}_{2}\right)$ that $C^{m}(\bar{G})$ is dense in $W^{m, 2}(G)$ (cf. Lions [11], p. 208). Let $B$ be the boundary of $G$ and $V_{0}=\sum_{|\beta| \leqq m} \oplus\left(L_{2}(B)\right)_{\beta}$, where for each multi-index $\beta$ the corresponding summand is a replica of $L^{2}(B)$ formed with respect to integration in surface area. We define a mapping $\zeta$ of $C^{m}(\bar{G})$ into $V_{0}$ by setting $(\zeta u)_{\beta}=\left.D^{\beta} u\right|_{B}$. It follows from $\left(\mathrm{g}_{2}\right)$ that $\zeta$ is a bounded linear mapping of the dense subset $C^{m}(\bar{G})$ of $W^{m, 2}(G)$ into $V_{0}$ and hence extendable to a bounded linear mapping of $W^{m, 2}(G)$ into $V_{0}$ which we shall also designate as $\zeta$. Furthermore $\zeta u=0$ if and only if $u \varepsilon \dot{W}^{m, 2}(G)$ (Schwartz [14]).

For the finite-dimensional subspace $F_{0}$ of $W^{m, 2}(G)$ defined above, $\zeta\left(F_{0}\right)$ is a finite-dimensional and hence closed subspace of $V_{0}$. If $V_{0}^{\prime}=V_{0} \ominus \zeta\left(F_{0}\right)$, $V_{0}^{\prime}$ is a closed linear subspace of $V_{0}$. If $u \varepsilon W^{m, 2}(G)$, the projection of $\zeta u$ on $V_{0}^{\prime}$ equals $\zeta u$ - $\zeta f$, where $f$ is some element of $F_{0}$ and hence lies in $\zeta\left(W^{m, 2}(G)\right)$. Thus $\zeta\left(W^{m, 2}(G)\right)=\zeta\left(F_{0}\right)+\zeta\left(W^{m, 2}(G)\right) \cap V_{0}^{\prime}$. We set $W_{1}=\zeta^{-1}\left(V_{0}^{\prime}\right)$. Since $(\zeta u, \zeta f)_{v_{0}}$ for each fixed $f$ in $F_{0}$ is a continuous linear functional of $u$ in $W^{m, 2}(G)$, $W_{1}$ is a closed subspace of $W^{m, 2}(G)$. Further, $W^{m, 2}(G)=W_{1}+F_{0}$.

Lemma 2: The inner product $a(u, v)$ on $W_{1}$ yields a norm equivalent to the $W^{m, 2}$-norm. 
Proof of Lemma 2: Since $W_{1}$ has no elements in common with $F_{0}, a(u, u)>0$ for $u \varepsilon W_{1}$. Suppose that Lemma 2 were false. Then there must exist a sequence $u_{r}$ with $u_{r} \varepsilon W_{1}$ such that $\left\|u_{r}\right\|=1$ while $a\left(u_{r}, u_{r}\right) \rightarrow 0$ as $r \rightarrow \infty$. By $\left(\mathrm{p}_{2}\right)$ it follows that $\left\|u_{r}\right\|_{m} \rightarrow 0$ as $r \rightarrow \infty$. Choosing a subsequence by $\left(\mathrm{g}_{1}\right)$, we may assume that $\left\{u_{r}\right\}$ is a Cauchy sequence in $W^{m-1,2}(G)$. But then

$$
\left\|u_{r}-u_{s}\right\|^{2} \leqq \sum_{j<m}\left\|u_{r}-u_{s}\right\|_{i}^{2}+\left(\left\|u_{r}\right\|_{m}+\left\|u_{s}\right\|_{m}\right)^{2} \rightarrow 0
$$

as $r, s \rightarrow \infty$, so that $\left\{u_{r}\right\}$ is a Cauchy sequence in $W^{m, 2}(G)$. Hence $u_{r}$ converges in $W^{m, 2}(G)$ to some function $v$ from $W^{m, 2}(G)$ and $\|v\|=\lim _{r}\left\|u_{r}\right\|=1$. Since $W_{1}$ is closed and the sequence lies in $W_{1}, v \varepsilon W_{1}$. On the other hand, $\|u\|_{m}$ is a continuous function of $u$ in $W^{m, 2}(G)$, so that $\|v\|_{m}=\lim _{r}\left\|u_{r}\right\|_{m}=0$ and $v \varepsilon F_{0}$. Since $W_{1} \cap F_{0}=\{0\}, v=0$ which contradicts the fact that $\|v\|=1$. Thus the lemma must be true.

A particular consequence of Lemma 2 is that $W_{1}$ is a Hilbert space with respect to the inner product $a(u, v)$. If we specialize the space $F$ of Theorem 1 to be our Hilbert space $W_{1}$ with inner product $a(u, v)$ and repeat the argument of Theorem 2, we obtain

Theorem 3: There is an unique function $k(x, y) \varepsilon C^{\infty}(G \times G)$ which is the kernel function for $W_{1}$ in the sense of Theorem 1 . Moreover, if $\psi_{i}(x)$ are a complete orthonormal sequence in $W_{1} \cap H$ with respect to the inner product $a(u, v)$, then

$$
k(x, y)=\sum_{j=1}^{\infty} \overline{\psi_{i}(x)} \psi_{j}(y)
$$

the series converging uniformly on compact subsets of $G \times G$ together with all partial derivatives.

Let $G_{1}$ be an open subset of $G$ and $J_{G_{1}}$ the natural projection of $W^{m, 2}(G)$ into $W^{m, 2}\left(G_{1}\right)$ with $J_{G_{2}} u$ defined to be the restriction of $u$ to $G_{1}$. Let $K_{G_{2}}$ be the norm-preserving mapping of $\dot{W}^{m, 2}\left(G_{1}\right)$ into $\dot{W}^{m, 2}(G)$ obtained by extending by continuity the identity mapping of $C_{c}^{\infty}\left(G_{1}\right)$ into $C_{c}^{\infty}(G)$. Thus $K_{G_{1}} u$ is the element of $\dot{W}^{m, 2}(G)$ obtained from $u$ by setting $u$ equal to zero outside $G_{1}$. Finally, let $Q_{G_{1}}$ be the mapping of $W^{m, 2}\left(G_{1}\right)$ into itself defined by letting $Q_{G_{1}} u$ be the solution of $A v=0$ in $G_{1}$ having the same Dirichlet data on the boundary of $G_{1}$ as $u$. We remark that if $A$ satisfies $\left(\mathrm{p}_{2}\right)$ on $G$, the Dirichlet problem is uniquely solvable on every bounded open subset $G_{1}$ of $G$. In particular, if $G$ satisfies $\left(\mathrm{g}_{2}\right)$, it is bounded and the Dirichlet problem on $G$ is uniquely solvable. Let $P u$ denote, as before, the solution of the equation $A v=0$ in $G$ having the same Dirichlet data as the given function $u$ in $W^{m, 2}(G)$.

If $u \varepsilon W^{m, 2}(G), J_{G_{1}} u$ is its restriction to the subset $G_{1}$ and $Q_{G_{1}}\left(J_{G_{1}} u\right)$ is the solution of $A v=0$ on $G_{1}$ having the same Dirichlet data on the boundary of $G_{1}$ as $J_{G_{1}} u$. Then the function $J_{G_{2}} u-Q_{G_{1}} J_{G_{2}} u$ lies in $\dot{W}^{m, 2}\left(G_{1}\right)$. Its extension to $G$, setting it equal to zero outside $G_{1}$, is $g=K_{G_{1}}\left(J_{G_{1}} u-Q_{G_{1}} J_{G_{1}} u\right)$. Finally, $v=P_{a_{1}} u=u-g$ is the function from $W^{m, 2}(G)$ which is equal to $u$ outside 
$G_{1}$ and which inside $G_{1}$ is the solution of the equation $A v=0$ having the same Dirichlet data as $u$ on the boundary of $G_{1} . P_{G_{1}}$ for each open subset $G_{1}$ of $G$ is a bounded idempotent linear mapping of $W^{m, 2}(G)$ into itself.

Let us note that $P_{G_{1}} u=u-g$, where $g \varepsilon \dot{W}^{m, 2}(G)$. As a consequence, $\zeta P_{G_{1}} u=\zeta u$ for all $u$ in $W^{m, 2}(G)$. In particular, if $u \varepsilon W_{1}$, then $P_{G_{1}} u \varepsilon W_{1}$ for any open subset $G_{1}$ of $G$.

We shall construct a new inner product on $W^{m, 2}(G)$ which yields a Hilbert space structure on that set with norm equivalent to the $W^{m, 2}$-norm and in which $P_{G_{1}}$ is an orthogonal projection for every open subset $G_{1}$ of $G$. By our previous remarks and Lemma $2, W^{m, 2}(G)=W_{1}+F_{0}$, where $W_{1}$ is a closed subspace of $W^{m, 2}(G)$ and the inner product $a(u, v)$ on $W_{1}$ yields a norm equivalent to $\|\cdot\|$.

Let $u=w_{1}+f, v=w_{2}+g$, where $w_{1}, w_{2} \varepsilon W ; f, g \varepsilon F_{0}$. We define the inner product $\langle u, v\rangle=a\left(w_{1}, w_{2}\right)+[f, g]=a(u, v)+[f, g]$. Note that $\langle u, u\rangle=$ $a\left(w_{1}, w_{1}\right)+[f, f] \geqq 0$ and $\langle u, u\rangle=0$ if and only if $u=0$. Furthermore, $W^{m, 2}(G)$ is complete with respect to the norm $\|u\|=\langle u, u)^{\frac{1}{2}}$, for if $\left\{u_{r}\right\}\left(u_{r}=w_{r}+f_{r}\right.$, $w_{r} \varepsilon W_{1}, f_{r} \varepsilon F_{0}$ ) form a Cauchy sequence with respect to this norm, then $\left\{w_{r}\right\}$ and $\left\{f_{r}\right\}$ are Cauchy sequences with respect to the $W^{m, 2}$-norm and therefore converge in that norm to elements $w_{0}$ and $f_{0}$ of $W_{1}$ and $F_{0}$, respectively, since $W_{1}$ and $F_{0}$ are closed subspaces of $W^{m, 2}(G)$. It follows that $u_{r}$ converges to $w_{0}+f_{0}$ in the new norm.

We shall now verify that $P_{a_{2}}$ is an orthogonal projection with respect to this new inner product. Let us remark first that $P_{G_{1}}$ is the identity operator on $F_{0}$ since $F_{0}$ is contained in $H$. Note next that for any $v$ in $W^{m, 2}(G), v=P_{G_{1}} v+h_{1}$ where $h_{1}$ is the limit in $W^{m, 2}(G)$ of a sequence $\left\{\phi_{r}\right\}$ with $\phi_{r} \varepsilon C_{c}^{\infty}\left(G_{1}\right)$. In particular, if $u$ is any element of $W^{m, 2}(G), a\left(P_{G_{2}} u, h_{1}\right)=\lim _{r} a\left(P_{G_{1}} u, \phi_{r}\right)=0$, since $P_{G_{1}} u$ is a solution of $A v=0$ in $G_{1}$. Therefore $a\left(P_{G_{1}} u, v\right)=a\left(P_{G_{1}} u, P_{G_{2}} v\right)$. Finally, $\left\langle P_{G_{1}} u, v\right\rangle$, for $u=w_{1}+f, v=w_{2}+g$ as above, equals $a\left(P_{G_{1}} u, v\right)+[f, g]$. Thus $\left\langle P_{G_{1}} u, v\right\rangle=a\left(P_{G_{1}} u, P_{G_{1}} v\right)+[f, g]=\left\langle u, P_{G_{1}} v\right\rangle$.

Let $G_{j}$ be a sequence (finite or infinite) of open subsets of $G, 1 \leqq j \leqq J \leqq \infty$. Suppose that $\left\{G_{i}\right\}$ forms a covering of $G$. Let $\pi$ be an infinite sequence of positive integers $\left(n_{1}, n_{2}, \cdots\right)$ with $1 \leqq n_{k} \leqq J$.

Definition 6: The sequence $\pi$ is said to be a permissible sequence if for each $j$ with $1 \leqq j \leqq J$ there exists an integer $K_{j}$ such that every block of $K_{i}$ successive indices $n_{k}$ includes the integer $j$ at least once.

It is easy to verify that for each $J$, finite or infinite, there are permissible sequences of $J$ indices. A very important special case is that of $J=2$ and the sequence $\pi$ consisting of alternating 1's and 2's. Let $P_{i}=P_{G_{i}}, P=P_{G}$.

Theorem 4: Let $u$ be a given function in $W^{m, 2}(G)$ and let $R_{s} u=P_{n}, P_{n,-1} \ldots$ $P_{n_{2}} P_{n_{1}} u$ for a given permissible sequence $\pi$. Then:

(a) $R_{s} u$ converges weakly to $P u$ in $W^{m, 2}(G)$ as $s \rightarrow \infty$. 
(b) $R_{s} u$ converges strongly to $P u$ in $W^{m-1,2}(G)$ as well as in $L^{p}(G)$ with $p=$ $n /(n-2 m)$ for $2 m<n$, all $L^{p}(G)$ for $n=2 m$, and in $C^{s}(\bar{G})$ if $m+s>\frac{1}{2} n$.

(c) Let $\dot{G}_{j}$ be the boundary of $G_{i}, G^{\prime}=G-\bigcup_{i} \dot{G}_{i}$. Then $R_{s} u$ converges uniformly to $P u$ on every compact subset of $G^{\prime}$ together with all partial derivatives.

(d) If $J=2$ and $\pi$ is the alternating sequence, $R_{\mathrm{\imath}} u$ converges strongly to $P u$ in $W^{m, 2}(G)$.

Remark on Theorem 4: The sequence $R_{s}$ for the alternating sequence when $J=2$ is that of the Schwarz alternating process; the more general sequence $R_{s}$ for an arbitrary permissible sequence yields a generalization of the Poincaré balayage process.

Proof of Theorem 4: (b) follows from (a), together with theorems of Soвolev and Kondrashov on the imbedding of $W^{m, 2}(G)$ in $L^{p}, C^{s}(\bar{G})$ (Sobolev [15], KondRASHOv [10]). Note that $\left(\mathrm{g}_{1}\right)$ shows that weak convergence in $W^{m, 2}(G)$ implies strong convergence in $W^{m-1,2}(G)$. (c) follows directly from (a) and (b) since every compact subset $K$ of $G^{\prime}$ can be decomposed into a finite number of subsets $K_{b}$ each contained in some $G_{i}$ and for $s \geqq s_{0}$, for some $s_{0}$, all $R_{s} u$ will be solutions of the equation $A v=0$ on some fixed neighborhood of each $K_{h}$.

Since $P_{1}$ and $P_{2}$ are orthogonal projections with respect to our new inner product, (d) is equivalent to a well known theorem of RELLICH and voN NEUMANN. Note that $R_{2 s+1}=\left(P_{1} P_{2} P_{1}\right)^{8}$ where $P_{1} P_{2} P_{1}$ is self-adjoint and $0 \leqq$ $P_{1} P_{2} P_{1} \leqq I$. It follows easily that $R_{2 s+1} u$ is strongly convergent. Since $R_{2 s} u=$ $P_{2} R_{2 s-1} u, R_{2 s} u$ is strongly convergent to a limit $w$. Let $v$ be the strong limit of $R_{2 s+1} u$. Then $P_{2} v=w, P_{1} w=v$ implies $\|w\| \leqq\|v\| \leqq\|w\|$ and the equality of norms implies $v=w, P_{1} v=P_{2} v=v$.

(a) follows from the following lemma whose proof is due to S. KakUtanI. KAKUTANI's original proof considered only a finite number of distinct projections, and we have made a slight alteration to allow for an infinite number of different $P_{j}$.

Lemma 3: Let $\left\{P_{i}\right\}$ be a sequence of orthogonal projections in a Hilbert space $W$, $\pi$ a permissible sequence of indices, $R_{s}=P_{n}, P_{n_{s-1}} \cdots P_{n_{2}} P_{n_{1}}, M_{i}$ the range of $P_{i}, M_{0}$ the intersection of all $M_{i}$, and $P_{0}$ the projection on $M_{0}$. Then $R_{s} u$ converges weakly in $W$ to $P_{0} u$ as $s \rightarrow \infty$.

Proof of Lemma 3: Since $M_{0}$ is contained in $M_{j}$ for every $j, P_{j} M_{0}=M_{0}$ and $P_{i}\left(W \ominus M_{0}\right) \subset W \ominus M_{0}$. Since $R_{s}$ is the identity on $M_{0}$, it suffices to show that $R_{s} u$ converges weakly to zero if $u \varepsilon W \ominus M_{0}$. Thus we may assume $M_{0}=\{0\}$.

Let $u_{s}=R_{s} u$. Since $u_{s-1}-u_{s}=\left(I-P_{n_{s}}\right) u_{s-1}$ is orthogonal to $u_{s}=P_{n} u_{s-1}$, $\left\|u_{s-1}-u_{s}\right\|^{2}=\left\|u_{s-1}\right\|^{2}-\left\|u_{s}\right\|^{2}$. Summing, we see that $\sum_{s}\left\|u_{s-1}-u_{s}\right\|^{2} \leqq\|u\|^{2}$ and as a consequence $\left\|u_{s-1}-u_{s}\right\| \rightarrow 0$ as $s \rightarrow \infty$. Iterating the argument, for every fixed integer $r,\left\|u_{s+r}-u_{s}\right\| \rightarrow 0$ as $s \rightarrow \infty$.

Since $\left\|u_{s}\right\| \leqq\|u\|$, by the compactness of closed balls in Hilbert space, it suffices to show that if $\left\{u_{s_{k}}\right\}$ is a weakly convergent subsequence of the sequence 
$u_{s}$ with limit $v$, then $v=0$. Let $j$ be an arbitrary integer. If $u_{s_{k}}$ converges weakly to $v$ as $k \rightarrow \infty$, then, by the conclusion of the preceeding paragraph, so does $u_{s_{k+r}}$ for $0 \leqq r \leqq K_{i}$. So does any subsequence obtained by interspersing this finite family of subsequences. For each $k$ choose $r_{k}, 0 \leqq r_{k} \leqq K_{j}$, so that $n_{s_{k+r_{k}}}=j$. Then $u_{s_{k+r_{k}}}$ converges weakly to $v$ as $k \rightarrow \infty$. But $u_{s_{k+r_{k}}} \varepsilon M_{j}$, and since $M_{j}$ is a closed subspace, it contains all weak limits of sequences from $M_{j}$. Thus $v \varepsilon M_{i}$. Since $j$ was arbitrary, $v \varepsilon M_{0}$ and since by assumption $M_{0}=\{0\}$, $v=0$.

\section{BiBLIOGRAPHY}

[1] Bergman \& M. Schiffer, Kernel functions and elliptic differential equations in mathematical physics, New York, 1953.

[2] F. E. BrowDER, (a) The Dirichlet problem for linear elliptic equations of arbitrary order, Proc. Nat. Acad. Sci. 38 (1952) pp. 230-235; (b) The Dirichlet and vibration problems for linear elliptic differential equations of arbitrary order, Proc. Nat. Acad. Sci. 38 (1952) pp. 741-747; (c) Assumption of boundary values and the Green's function in the Dirichlet problem for the general linear elliptic equation, Proc. Nat. Acad. Sci. 39 (1953) pp. 179-184; (d) Linear parabolic differential equations of arbitrary order; general boundary value problems for elliptic equations, Proc. Nat. Acad. Sci. 39 (1953) pp. 185-190; (e) On the eigenfunctions and eigenvalues of the general linear elliptic differential operator, Proc. Nat. Acad. Sci. 39 (1953) pp. 433-439.

[3] F. E. Browder, Strongly elliptic systems of differential equations, Ann. Math. Study No. 33, Princeton, 1954, pp. 15-51.

[4] F. E. Browber, On the regularity properties of solutions of elliptic differential equations, Comm. Pure App. Math. 9 (1956) pp. 351-361.

[5] F. E. Browder, General boundary-value problems for partial differential equations, to appear.

[6] R. Courant \& D. Hilbert, Methoden der matematischen Physik, v. 2, Berlin, 1937.

[7] K. O. FrIEDRICHS, On the differentiability of solutions of linear elliptic differential equations, Comm. Pure App. Math. 6(1953) pp. 299-325.

[8] L. Gi̊ndng, Dirichlet's problem for linear elliptic partial differential equations, Math. Scand. 1 (1953) pp. 55-72.

[9] O. V. Guseva, On boundary problems for strongly elliptic systems, Doklad. Akad. Nauk 102 (1955) pp. 1069-1072.

[10] V. I. Kondrashov, On some properties of functions in space, Doklad. Akad. Nauk 57 (1947) pp. 111-114.

[11] J. L. Lions, Sur les problèmes aux limites du type dérivée oblique, Ann. of Math. 64 (1956) pp. 207-239.

[12] L. Nirenberg, Remarks on strongly elliptic partial differential equations, Comm. Pure App. Math. 8 (1955) pp. 643-674.

[13] L. Schwartz, Theorie des distributions, v. 2, Paris, 1951.

[14] L. Schwartz, Seminar notes, C.N.R. Paris, 1954-55.

[15] S. Sobolev, On a theorem of functional analysis, Mat. Sbornik 4 (1938) pp. 471-497.

[16] M. I. VIsIK., On strongly elliptic systems of differential equations, Mat. Sbornik 29 (1951) pp. 615-676.

Yale University

New Haven, Connecticut 